A14

doi: 10.14232/ syrpharmacognosy.2021.a14

\title{
Semi-synthetic preparation and investigation of new, potentially bioactive ecdysteroid derivatives
}

Dávid Laczkó

Email: LaczkoDavid@szte.hu

Ecdysteroids are a group of bioactive, non-toxic compounds known as analogues of the insect molting hormone. Some of their derivatives have been shown to have antidiabetic [1], cholesterol-lowering [2], immunomodulatory [3], antioxidant and neuroprotective [4] effects, and a recent in silico study suggested anti-COVID19 activity of an ecdysteroid [5]. Further, the tumor resistance-reducing effect of ecdysteroids was discovered by the research group that hosts my work [6].

The aim of our present research is to identify new bioactive ecdysteroid derivatives. Our experimental efforts include the methodological implementation of large-scale semi-synthetic production and purification of calonysterone that has a diverse pharmacological potential but limited availability [7]. It is also our objective to prepare further derivatives of this compound.

To this, end, we have performed the functionalization of calonysterone through an oxime functional group that is a valuable building block in organic syntheses, and its presence can directly affect the bioactivity of compounds. Eleven new ecdysteroid derivatives were prepared so far. Their bioactivity studies are carried out in collaboration, and they are currently being tested for neuroprotective, anti-Trypanosoma cruzi, antidiabetic and antihyperlipidemic activities.

Supervisors: Attila Hunyadi, Máté Vágvölgyi

\section{Acknowledgements:}

This work was supported by the National Research, Development and Innovation Office, Hungary (NKFIH; K-134704), the NKFIH Cooperative Doctoral Program for Doctoral Scholarships (KDP-2020), the Economic Development and Innovation Operative Program GINOP-2.3.2-15-2016-00012, and the Ministry of Human Capacities, Hungary grant 203913/2018/FEKUSTRAT.

\section{References}

[1] Yoshida, T., et al. Biochem Pharmacol 1971; 20(12): 3263-3268.

[2] Yahya AI N., M. G., Kariman M. Am J Life Sci 2017; 5: 7-10.

[3] Trenin, D. S. and V. V. Volodin. Arch Insect Biochem Physiol 1999; 41(3): 156-161.

[4] Hu, J., et al. PLoS One 2012; 7(12): e50764.

[5] Mubarak A. Alamri, et al., Arab J Chem 2020; 13: 7224-7234

[6] Vagvolgyi M., et al, Eur J Med Chem 2018; 144: 730-739.

[7] Issaadi HM, et al., J Chromatogr B 2017; 1054: 44-49 